Gazi University
Journal of Science
$\mathrm{http} / /$ dergipark.gov.tr/gujs

\title{
A Comparison Analysis of Fuzzy and Bayesian Linear Model Parameter Estimates for Replicated Response Measures
}

\author{
Ozlem TURKSEN* \\ Ankara University, Department of Statistics, 06100, Ankara, Turkey.
}

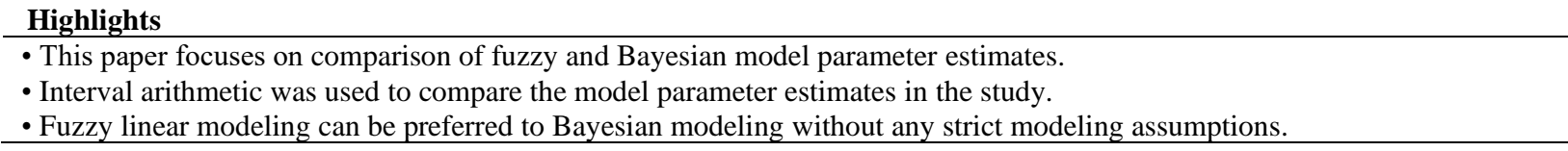

\section{Article Info}

Received: $17 / 12 / 2019$

Accepted: 05/07/2020

\section{Keywords}

Replicated response measures,

Fuzzy modeling

Bayesian modeling

Interval arithmetic

\begin{abstract}
It is possible to define functional relationship between replicated response measures and input variables by using fuzzy and Bayesian modeling approaches. The main aim of the study is to present the alternative usability of fuzzy modeling approach to Bayesian modeling approach with defining a proper alpha-cut level among the many alpha-cut levels. In this study, the uncertainty of estimated model parameters were compared by transforming the estimated parameter values to intervals. Interval valued parameter estimates were obtained through alpha-cut level presentation and credible intervals for fuzzy and Bayesian approaches, respectively. Thus, it was achieved to model the replicated response measured (RRM) data set without making any probabilistic modeling assumptions which were hard to satisfy for small sized RRM data set. To compare the interval valued model parameter estimates in the proposed study, midpoint, width, radius and Hausdorff metrics were used. And also, interval type residuals were calculated to see the performance of predicted fuzzy and Bayesian models for making clear comparison. Two data sets from the literature, which were called Roman Catapult and Printing Ink, were used and the obtained results were discussed in application part.
\end{abstract}

\section{INTRODUCTION}

Experimental designs are commonly used in many fields of science before composing a predicted model of a response of interest and a number of associated input variables. In some cases, experimenters need to measure the response values with the same input levels in different experimental runs, which is called replication, to increase the precision of the predicted model. Analysis of replicated response measured (RRM) data set enables to examine multiple source of variability, e.g. changing equipment settings, environmental factors, which cause experimental error. In general, the unknown relationship between the variables is approximated by a low-degree polynomial statistical model in which the error is assumed to be random and the model parameters are crisp [1]. However, the unknown model parameters should have flexible structure for modeling of the RRM data set since the qualification of the replicated measures has uncertainty. It is clear here that the replicated response measures cannot be exactly represented with a single numerical quantity. In this case, fuzzy and Bayesian modeling approaches can be used alternatively from possibilistic and probabilistic modeling perspective, respectively.

In the literature, it is possible to see the fuzzy approach applications for response modeling due to its ability about defining uncertainty of the data sets. Fuzzy set theory, firstly introduced by [2], helps to analyse the uncertainty by the use of fuzzy numbers with a membership function. In response surface studies, one of 
the common way of expressing uncertainty with fuzzy approach is using fuzzy regression at modeling stage of the data set. The fuzzy regression model was used for an off-line quality engineering problem for the first time by [3]. Fuzzy approach was applied for modeling of dual responses in the study of [4]. A new response surface method was proposed by using fuzzy logic models by [5]. In [6], the application of fuzzy logic and gave comparative results with classical modeling approach for the development of sugarcane juice was presented. Fuzzy least squares (FLS) approach was applied to response surface problems by using triangular type-1 fuzzy numbers (TT1FNs) in the study of [7]. And also, application of fuzzy modeling approach to RRM data set through TT1FNs can be seen in the studies of [8-12].

There have been several studies about Bayesian approach for response modeling. A Bayesian approach for empirical regression modeling was presented in [13]. Bayesian hierarchical approach was used to model dual response system by [14-16]. Bayesian predictive approach for multiresponse problem was applied by [17]. The application of Bayesian approach to the robust parameter design problem of multiresponse was presented in the study of [18]. Bayesian methods in response surface methodology in the context of "offline quality" improvement was used by [19]. Even there have been some studies about Bayesian approach for response surface modeling in the literature, there is only a work for application of Bayesian approach to RRM data set, studied by [11].

The main aim of this study is to present the alternative usability of the fuzzy approach to the Bayesian approach for modeling of RRM data set. The model parameters were assumed as random variables and TT1FNs for Bayesian and fuzzy modeling approaches, respectively. In this study, interval valued parameter estimates were obtained to make comparison for the uncertainty of estimated model parameters on the same domain. There have been several studies about application of interval type uncertainty. A new reliability estimation model based on the level cut strategy and volume ratio theory was proposed by [20] in the case that interval and fuzzy uncertainties exist simultaneously. [21] presented a non-probabilistic multidisciplinary uncertainty analysis method to obtain the bounds of system output variables when the uncertain variables were described as interval variables. A new analytic method of time-dependent reliability based on theory of non-probabilistic interval process for cracked structures with limited uncertainty information was proposed by [22].

In this study, interval indication of model parameter estimates was achieved by using alpha-cut level set presentation and highest posterior density (HPD) credible interval related to fuzzy approach and Bayesian approach, respectively. The purpose of the study was proposing an alternative flexible model, based on interval type fuzzy regression, instead of using Bayesian modeling for several situations, e.g. small sized RRM data sets, unsatistified probabilistic modeling assumptions. This was achieved by choosing a proper alpha-cut level among some alpha-cut levels in terms of similarity of interval valued Bayesian model parameter estimates. Several comparison metrics, e.g. midpoint, width, radius and Hausdorff, were used to make decision. Among them, the Hausdorff-metric is a well-known distance metric to compare the similarity of two closed intervals through midpoints and radius metrics. Detailed information about Hausdorff-metric and interval data can be seen in the study of [23, 24].

The paper was organized as follows. The fuzzy and Bayesian linear modeling approaches for RRM experiments were presented in section 2. In section 3, interval valued parameter estimates and comparison metrics were defined in detail. The applicability of preferable approach for fuzzy modeling was illustrated with the data sets from the literature in section 4 . Conclusions with comparison results were given in section 5 .

\section{FUZZY AND BAYESIAN LINEAR MODELING APPROACHES FOR REPLICATED RESPONSE MEASURES}

Composing a proper experimental design should be considered as a basic necessary step to get most valuable information about a modeling problem. In some cases, the researcher needs replicated measures of response variables for each experimental unit (observation). So, the experimental design is composed with replicated response measures as given in Table 1. 
Table 1. Experimental design with replicated response measures

\begin{tabular}{ccccccccc}
\hline & \multicolumn{3}{c}{ Input levels } & \multicolumn{4}{c}{$\underline{\text { Response }}$} \\
\cline { 2 - 9 } & $\mathbf{X}_{1}$ & $\mathbf{X}_{2}$ & $\ldots$ & $\mathbf{X}_{p}$ & \multicolumn{4}{c}{$\mathbf{Y}$} \\
\hline $\mathbf{1}$ & $x_{11}$ & $x_{12}$ & $\ldots$ & $x_{1 p}$ & $y_{11}$ & $y_{12}$ & $\ldots$ & $y_{1 r}$ \\
\hline $\mathbf{2}$ & $x_{21}$ & $x_{22}$ & $\ldots$ & $x_{2 p}$ & $y_{21}$ & $y_{22}$ & $\ldots$ & $y_{2 r}$ \\
\hline$\cdot$ &. &. & &. &. &. & & \\
$\cdot$ &. &. & $\ldots$ &. &. &. & $\ldots$ & \\
. &. &. & &. &. &. & & \\
\hline $\mathbf{n}$ & $x_{n 1}$ & $x_{n 2}$ & $\ldots$ & $x_{n p}$ & $y_{n 1}$ & $y_{n 2}$ & $\ldots$ & $y_{n p}$ \\
\hline
\end{tabular}

In Table $1, n$ denotes the number of experimental units and $r$ is the number of replications for each response. It should be noted that replications are measured for each setting of a group of $p$ input variables. The main purpose of creating an experimental design as given in Table 1 is obtaining much more accurate estimates with measuring the quantity of uncertainty for the response.

A modeling methodology of the RRM data set, given in Table 1, can be considered as linear regression model which is well-known and commonly used one. General form of the linear regression model, used in this study, can be given in a matrix form as

$\mathbf{Y}=\mathbf{X} \boldsymbol{\beta}+\boldsymbol{\varepsilon}$,

in which $\mathbf{Y}=\left[\begin{array}{lll}Y_{1} & Y_{2} \ldots Y_{n}\end{array}\right]^{\prime}$ is a response vector with $n$ observations $(n \times 1), \boldsymbol{\beta}=\left[\beta_{0} \beta_{1} \ldots \beta_{p}\right]^{\prime}$ is a vector of unknown model parameters $((p+1) \times 1), \quad \boldsymbol{\varepsilon}=\left[\varepsilon_{1} \varepsilon_{2} \ldots \varepsilon_{n}\right]^{\prime}$ is an error vector with independent and identically distributed (i.i.d.) random vectors $(n \times 1)$, and $\mathbf{X}$ is a matrix of settings of the input variables $(n \times(p+1))$. It should be noted here that the assumptions on $\varepsilon$ are zero mean and common variance.

Although the model given in Equation (1) is well-used one, the application of the model to RRM data set will need some adaptations. It is clear that representing of replicated measures with a single quantity will cause loss of information for each unit. Therefore, it is necessary to use modeling approaches with conserving natural data structure of replications which may contain uncertainty different than randomness.

In this study, two different types of modeling approaches, fuzzy and Bayesian, are used to estimate the unknown model parameters, $\boldsymbol{\beta}$, for RRM data set.

\subsection{Fuzzy Linear Modeling Approach}

Fuzzy modeling is considered to be one of the proper modeling approach for RRM data set due to its ability of dealing with the uncertainties of the nature of the replicated measures. In order to apply fuzzy linear modeling approach to unknown response, the linear model given in Equation (1) can be written as

$$
\tilde{\mathbf{Y}}=\mathbf{X} \tilde{\boldsymbol{\beta}}+\tilde{\boldsymbol{\varepsilon}}
$$

where the observed response, model parameters and error vector are assumed as fuzzy numbers denoted as $\tilde{\mathbf{Y}}=\left[\begin{array}{llll}\tilde{Y}_{1} & \tilde{Y}_{2} & \ldots & \tilde{Y}_{n}\end{array}\right]^{\prime}, \tilde{\boldsymbol{\beta}}=\left[\begin{array}{llll}\tilde{\beta}_{0} & \tilde{\beta}_{1} \ldots \tilde{\beta}_{p}\end{array}\right]^{\prime}$ and $\boldsymbol{\varepsilon}=\left[\begin{array}{llll}\tilde{\varepsilon}_{1} & \tilde{\varepsilon}_{2} & \ldots & \tilde{\varepsilon}_{n}\end{array}\right]^{\prime}$, respectively. Throughout the paper TT1FNs are preferred to use for the sake of simplicity. Here, the input variables are considered to be crisp. 
To present the observed replicated response measures as TT1FNs, some of the descriptive statistics of the replicated response measures are calculated. Therefore, fuzzification of replicates is achieved by taking into account the data structure of replications in statistical framework.

Let the matrix of observed replicated response measures be

$\mathbf{y}=\left[\begin{array}{c}\mathbf{y}_{1} \\ \mathbf{y}_{2} \\ \cdot \\ \cdot \\ \cdot \\ \mathbf{y}_{n}\end{array}\right]=\left[\begin{array}{cccc}y_{11} & y_{12} & \ldots & y_{1 r} \\ y_{21} & y_{22} & \ldots & y_{2 r} \\ \cdot & \cdot & & \cdot \\ \cdot & \cdot & \ldots & \cdot \\ \cdot & \cdot & & \cdot \\ y_{n 1} & y_{n 2} & \ldots & y_{n r}\end{array}\right]$.

The TT1FN representations for each unit is given as

$\mathbf{y}=\left[\begin{array}{c}\tilde{\mathbf{y}}_{1} \\ \tilde{\mathbf{y}}_{2} \\ \cdot \\ \cdot \\ \cdot \\ \tilde{\mathbf{y}}_{n}\end{array}\right]=\left[\begin{array}{c}\left(y_{1}^{l}, y_{1}^{c}, y_{1}^{u}\right) \\ \left(y_{2}^{l}, y_{2}^{c}, y_{2}^{u}\right) \\ \cdot \\ \cdot \\ \cdot \\ \left(y_{n}^{l}, y_{n}^{c}, y_{n}^{u}\right)\end{array}\right]$.

Here, each fuzzy response is denoted as $\tilde{\mathbf{y}}_{i}=\left(y_{i}^{l}, y_{i}^{c}, y_{i}^{u}\right), i=1,2, \ldots, n$. In the studies of $[10,11]$, replicated response measures were transformed to TT1FNs by using mean and standard deviation statistics of replicated measures. However, these statistics may not be sufficient to present the data structure of replications during fuzzification. Different type of fuzzification rules for presenting the replicated response measures as TT1FNs were given in [12]. It was seen that the fuzzification with descriptive statistics and golden ratio was the most proper one. Therefore, in this study, replicated response measures are fuzzified by using the rule in the study of [12] as following

$y_{i}^{l}=y_{i(1)}$

$y_{i}^{c}=y_{i(1)}+r_{i} \times \rho, \quad i=1,2, \ldots, n, j=1,2, \ldots, r$

$y_{i}^{u}=y_{i(r)}$

where $y_{i(1)}$ and $y_{i(r)}$ are the smallest and the largest order statistics for each unit, respectively. $r_{i}$ is called as the range and obtained by $r_{i}=y_{i(r)}-y_{i(1)}, i=1,2, \ldots, n . \rho$ is the golden ratio and taken equal to 0.618 .

After fuzzification of replicated response measures, it is aimed to obtain predicted fuzzy linear response model. In this case, the predicted fuzzy linear regression model is written as

$\hat{\tilde{\mathbf{Y}}}=\mathbf{X} \hat{\tilde{\boldsymbol{\beta}}}+\hat{\tilde{\mathbf{e}}}$

in which $\hat{\tilde{\mathbf{Y}}}, \hat{\tilde{\boldsymbol{\beta}}}$ and $\hat{\tilde{\mathbf{e}}}$ are predicted fuzzy response values, estimated fuzzy model parameters and predicted fuzzy errors, denoted as $\hat{\tilde{\mathbf{Y}}}=\left(\hat{\mathbf{Y}}^{l}, \hat{\mathbf{Y}}^{c}, \hat{\mathbf{Y}}^{u}\right), \hat{\tilde{\boldsymbol{\beta}}}=\left(\hat{\boldsymbol{\beta}}^{l}, \hat{\boldsymbol{\beta}}^{c}, \hat{\boldsymbol{\beta}}^{u}\right)$ and $\hat{\tilde{\mathbf{e}}}=\left(\hat{\mathbf{e}}^{l}, \hat{\mathbf{e}}^{c}, \hat{\mathbf{e}}^{u}\right)$, respectively. In order to obtain the estimates of triangular type fuzzy model parameters FLS approach is used. The FLS is based on minimizing the sum of the squared distance between observed and predicted fuzzy response values, formalized as 


$$
\min _{\tilde{\boldsymbol{\beta}}} \phi(\tilde{\boldsymbol{\beta}})=\min _{\tilde{\tilde{\boldsymbol{\beta}}}}(d(\tilde{\mathbf{Y}}, \hat{\tilde{\mathbf{Y}}}))
$$

where $d$ denotes the Diamond's distance metric, called vertex method, given in [25] as following

$$
d(\tilde{\mathbf{Y}}, \hat{\tilde{\mathbf{Y}}})=\sqrt{\frac{1}{3}\left(\left(\mathbf{Y}^{l}-\hat{\mathbf{Y}}^{l}\right)^{2}+\left(\mathbf{Y}^{c}-\hat{\mathbf{Y}}^{c}\right)^{2}+\left(\mathbf{Y}^{u}-\hat{\mathbf{Y}}^{u}\right)^{2}\right)} .
$$

The estimates of fuzzy model parameters are obtained by minimizing the Equation (7) and calculated as given below

$\hat{\tilde{\boldsymbol{\beta}}}=W \tilde{\mathbf{Y}}$

in which $W$ is equal to $\left(\mathbf{X}^{\prime} \mathbf{X}\right)^{-1} \mathbf{X}^{\prime}$. Assuming that the $\left(\mathbf{X}^{\prime} \mathbf{X}\right)^{-1}$ is nonsingular, matrix $W$ may have negative values. In this case, scalar multiplication should be done with considering the elementary operations rules of TT1FNs. Let matrix $W$ be

$W=\left[\begin{array}{cccc}w_{11} & w_{12} & \ldots & w_{1 n} \\ w_{21} & w_{22} & \ldots & w_{2 n} \\ \cdot & \cdot & & \cdot \\ \cdot & \cdot & \ldots & \cdot \\ \cdot & \cdot & & \cdot \\ w_{p+1,1} & w_{p+1,2} & \ldots & w_{p+1, n}\end{array}\right]_{(p+1) \times n}$

and the multiplication is

$$
W \tilde{\mathbf{Y}}=\left\{\begin{array}{cc}
\left(w_{i j} \mathbf{Y}^{l}, w_{i j} \mathbf{Y}^{c}, w_{i j} \mathbf{Y}^{u}\right) & , \quad w_{i j}>0 \\
\left(-w_{i j} \mathbf{Y}^{u},-w_{i j} \mathbf{Y}^{c},-w_{i j} \mathbf{Y}^{l}\right), & w_{i j}<0
\end{array} \quad, \quad i=1,2, \ldots, p+1, j=1,2, \ldots, n\right.
$$

where $w_{i j}$ is the element positioned in the $i$ th row and $j$ th column of matrix $W$. Therefore, according to the matrix operators given in Equation (11), the triangular fuzzy model parameter estimates, $\hat{\tilde{\boldsymbol{\beta}}}$, are obtained.

\subsection{Bayesian Linear Modeling Approach}

Bayesian can be considered another proper modeling approach for RRM data set since the ability of representing uncertainty of replicated response measures in the predicted model with assuming model parameters as random variables instead of crisp values. And also, Bayesian approach allows learning from the data with combining prior information of model parameters. Posterior distribution of unknown model parameters is called as Bayesian estimates of model parameters. Linear regression methods can be thought of as Bayesian posterior inference based on a non-informative prior distribution for the parameters of the normal linear model.

Consider the linear regression model given in matrix form as in Equation (1) and recall the assumptions concerning the elements of $\boldsymbol{\varepsilon}$ with Normally distributed, $\boldsymbol{\varepsilon} \sim N_{n}\left(0, \sigma^{2} I_{n}\right)$. It is clear that the model which relates parameters and observations is written as 


$$
\mathbf{Y} \mid \boldsymbol{\beta}, \sigma^{2}, \mathbf{X} \sim N_{n}\left(\mathbf{X} \boldsymbol{\beta}, \sigma^{2} I_{n}\right) .
$$

After defining the model, Bayesian analysis focuses to discover the posterior distribution for the parameters. The analysis begins with a prior distribution for the parameters. A non-informative prior distribution, commonly used for linear regression, can be defined as

$$
h\left(\boldsymbol{\beta}, \sigma^{2}\right) \propto\left(\sigma^{2}\right)^{-1}, \quad \boldsymbol{\beta} \in R^{p}, \quad \sigma^{2}>0 .
$$

The expression given in Equation (13) means that the joint probability distribution of $\boldsymbol{\beta}$ and $\sigma^{2}$ is a flat surface with a constant level proportional to $\frac{1}{\sigma^{2}}$. Applying Bayes Theorem, the posterior density of the $\boldsymbol{\beta}$ and $\sigma^{2}$, conditional on the data, is given by a proportion below [11]

$$
h\left(\boldsymbol{\beta}, \sigma^{2} \mid \mathbf{Y}\right) \propto\left(\sigma^{2}\right)^{-\left(\frac{k}{2}+1\right)} \cdot e^{-\frac{k S^{2}}{2 \sigma^{2}}} \times\left(\sigma^{2}\right)^{-\frac{p}{2}} \cdot e^{\left.-\frac{1}{2 \sigma^{2}}\left[(\boldsymbol{\beta}-\hat{\boldsymbol{\beta}}) \mathbf{x}^{\prime} \mathbf{x} \mathbf{x}-\hat{\boldsymbol{\beta}}\right)\right]} .
$$

While the posterior distribution of $\boldsymbol{\beta}$ given $\sigma^{2}$ and $\mathbf{Y}$ is

$$
\boldsymbol{\beta} \mid \sigma^{2}, \mathbf{Y} \sim N_{p+1}\left(\hat{\boldsymbol{\beta}}, \sigma^{2}\left(\mathbf{X}^{\prime} \mathbf{X}\right)^{-1}\right)
$$

and the marginal posterior distribution of $\boldsymbol{\beta}$ is multivariate Student-t distribution given as

$$
\boldsymbol{\beta} \mid \mathbf{Y} \sim t_{p+1}\left(k ; \hat{\boldsymbol{\beta}}, S^{2}\left(\mathbf{X}^{\prime} \mathbf{X}\right)^{-1}\right)
$$

where $\hat{\boldsymbol{\beta}}$ and $S^{2}$ are the maximum likelihood estimates of $\boldsymbol{\beta}$ and $\sigma^{2}$ parameters, respectively. Here, $k S^{2}=(\mathbf{Y}-\mathbf{X} \hat{\boldsymbol{\beta}})^{\prime}(\mathbf{Y}-\mathbf{X} \hat{\boldsymbol{\beta}}), \quad k=n-(p+1)$ and $\hat{\boldsymbol{\beta}}=\left(\mathbf{X}^{\prime} \mathbf{X}\right)^{-1} \mathbf{X}^{\prime} \mathbf{Y}$ [26]. Thus, the marginal posterior distribution of each regression model parameter $\beta_{j}, j=0,1,2, \ldots, p$ is obtained as

$$
\beta_{j} \mid Y \sim t_{1}\left(k ; \hat{\beta}_{j}, S^{2}\left(\mathbf{X}^{\prime} \mathbf{X}\right)_{j j}^{-1}\right) .
$$

\section{INTERVAL VALUED PARAMETER ESTIMATES}

Determining the uncertainty of model parameters through fuzzy and Bayesian approaches are distinct from each other. For this purpose, it is necessary to define a common uncertainty presentation for the model parameters. In this study, interval valued parameter estimates are obtained to present the fuzzy and Bayesian linear model parameters since transforming the model parameter estimates to intervals provides convenience for comparison of the parameters uncertainty on the same domain.

\subsection{Fuzzy alpha-cut level interval}

To define the fuzzy parameter estimates as crisp intervals, a well-known approach is representing the $\hat{\tilde{\boldsymbol{\beta}}}=\left(\hat{\boldsymbol{\beta}}^{l}, \hat{\boldsymbol{\beta}}^{c}, \hat{\boldsymbol{\beta}}^{u}\right)$ as a family of sets called alpha-level set. The alpha-level set of $\hat{\tilde{\boldsymbol{\beta}}}$ is defined as

$$
\hat{\tilde{\boldsymbol{\beta}}}=\left\{\hat{\boldsymbol{\beta}} \in R: \mu_{\hat{\hat{\boldsymbol{\beta}}}}(\hat{\boldsymbol{\beta}}) \geq \alpha\right\}
$$


where $\mu_{\hat{\boldsymbol{\beta}}}(\hat{\boldsymbol{\beta}})$ is identified as below

$$
\mu_{\hat{\hat{\beta}}}(\hat{\boldsymbol{\beta}})=\left\{\begin{array}{cc}
\frac{\hat{\boldsymbol{\beta}}-\hat{\boldsymbol{\beta}}^{l}}{\hat{\boldsymbol{\beta}}^{c}-\hat{\boldsymbol{\beta}}^{l}} \quad, \quad \hat{\boldsymbol{\beta}}^{l} \leq \hat{\boldsymbol{\beta}} \leq \hat{\boldsymbol{\beta}}^{c} \\
\frac{\hat{\boldsymbol{\beta}}^{u}-\hat{\boldsymbol{\beta}}}{\hat{\boldsymbol{\beta}}^{u}-\hat{\boldsymbol{\beta}}^{c}} \quad, \quad \hat{\boldsymbol{\beta}}^{c} \leq \hat{\boldsymbol{\beta}} \leq \hat{\boldsymbol{\beta}}^{u} . \\
0, & \text { o.w. } \\
&
\end{array}\right.
$$

It is possible to obtain a crisp interval of $\hat{\tilde{\boldsymbol{\beta}}}$ easily by alpha-cut operation, denoted with $\hat{\tilde{\boldsymbol{\beta}}}_{\alpha}$, as follows

$$
\begin{aligned}
\hat{\tilde{\boldsymbol{\beta}}}_{\alpha} & =[L(\alpha), U(\alpha)] \\
& =\left[\hat{\boldsymbol{\beta}}^{l}+\left(\hat{\boldsymbol{\beta}}^{c}-\hat{\boldsymbol{\beta}}^{l}\right) \alpha, \hat{\boldsymbol{\beta}}^{u}-\left(\hat{\boldsymbol{\beta}}^{u}-\hat{\boldsymbol{\beta}}^{c}\right) \alpha\right]
\end{aligned}
$$

in which $\alpha \in[0,1]$. It should be noted here that the smaller the value of alpha, the higher the uncertainty.

\subsection{Bayesian credible interval}

In order to define the Bayesian parameter estimates as crisp intervals, Bayesian credible interval is defined as a measure of the variation of model parameters. The Bayesian credible interval is similar to frequentist confidence interval and generally called as HPD credible interval.

In this study, HPD credible interval is used for analyzing the variation of each model parameters $\beta_{j}, j=0,1,2, \ldots, p$, defined as

$$
\left[\hat{\beta}_{j}-\sqrt{S^{2}\left(\mathbf{X}^{\prime} \mathbf{X}\right)_{j j}^{-1}} F_{t_{(k)}}^{-1}\left(\frac{1-\gamma}{2}\right), \hat{\beta}_{j}+\sqrt{S^{2}\left(\mathbf{X}^{\prime} \mathbf{X}\right)_{j j}^{-1}} F_{t_{(k)}}^{-1}\left(\frac{1-\gamma}{2}\right)\right]
$$

where $F_{t_{(k)}}^{-1}$ is a quantile of the Student-t posterior distribution with $(1-\gamma) \%$ confidence level.

\subsection{Comparison metrics for interval valued estimates of model parameters}

One of the main important aspect of the analysis of interval valued parameter estimates is the usage of convenient distance metric which is expected to be easy to calculate and interpret. In this study, midpoint, width, radius and the Hausdorff metrics are used for comparison purpose of the interval valued parameter estimates. More brief information on this topics can be seen in the study of [27, 28].

Let $\left[\beta_{i}\right]=\left[\beta_{i}^{-}, \beta_{i}^{+}\right], i=1,2, \ldots, p$, be an interval valued parameter estimates on $R$. The midpoint of $\left[\beta_{i}\right]$, $i=1,2, \ldots, p$, is defined as the real number

$$
\operatorname{mid}\left(\left[\beta_{i}\right]\right)=\frac{1}{2}\left(\beta_{i}^{-}+\beta_{i}^{+}\right), i=1,2, \ldots, p
$$

and the width and radius of $\left[\beta_{i}\right], i=1,2, \ldots, p$, are defined as, respectively,

$$
w\left(\left[\beta_{i}\right]\right)=\beta_{i}^{+}-\beta_{i}^{-}, i=1,2, \ldots, p
$$


and

$\operatorname{rad}\left(\left[\beta_{i}\right]\right)=\frac{1}{2} w\left(\left[\beta_{i}\right]\right)=\frac{1}{2}\left(\beta_{i}^{+}-\beta_{i}^{-}\right), i=1,2, \ldots, p$

Let $\left[\beta_{i}\right]=\left[\beta_{i}^{-}, \beta_{i}^{+}\right]$and $\left[\eta_{i}\right]=\left[\eta_{i}^{-}, \eta_{i}^{+}\right], i=1,2, \ldots, p$, be two intervals on $R$. Then, the Hausdorff-metric between $\left[\beta_{i}\right]$ and $\left[\eta_{i}\right]$ is given by

$H_{i}=H\left(\left[\beta_{i}\right],\left[\eta_{i}\right]\right)=\max \left\{\left|\beta_{i}^{-}-\eta_{i}^{-}\right|,\left|\beta_{i}^{+}-\eta_{i}^{+}\right|\right\}, i=1,2, \ldots, p$

The Equation (23) can also be expressed in terms of midpoints and radius:

$H_{i}=H\left(\left[\beta_{i}\right],\left[\eta_{i}\right]\right)=\left|\operatorname{mid}\left(\left[\beta_{i}\right]\right)-\operatorname{mid}\left(\left[\eta_{i}\right]\right)\right|+\left|\operatorname{rad}\left(\left[\beta_{i}\right]\right)-\operatorname{rad}\left(\left[\eta_{i}\right]\right)\right|, i=1,2, \ldots, p$.

The higher the quantity of $H\left(\left[\beta_{i}\right],\left[\eta_{i}\right]\right)$, the lower the similarity of $\left[\beta_{i}\right]$ and $\left[\eta_{i}\right]$. In this study, Hausdorff metric is used to compare the two interval valued parameter estimates since it considers midpoint and radius metrics together as given in Equation (26).

\section{APPLICATION}

In this section, two data sets, called Roman Catapult data set and Printing Ink data set, are used for application purpose. Even though, the data sets are previously used in the study of [11], these data sets are preferred to use to present the alternatively usability of fuzzy approach providing that the definition of proper alpha-cut levels of fuzzy parameter estimates.

Roman Catapult data set: Roman Catapult data set is originally used in the study of [29] for an example of dual response analysis. The catapult experiment is designed with three inputs (length - $X_{1}$, stop angle $X_{2}$, pivot height $-X_{3}$ ) and three replicated response measures by using second order central composite design. The RRM data set can be seen in the study of [11]. In order to apply fuzzy modeling to Roman Catapult data set, it is necessary to present the replicated response measures as TT1FNs. The fuzzification of response is achieved according to the data structure of replicated measures. In order to understand the data structure of the replicated measures, box-plots of the responses were plotted. The distribution of the replicated measures can be seen from the box-plots, given in Figure 1, for each unit. It's clear from Figure 1 that the replicated values have skewed distribution.

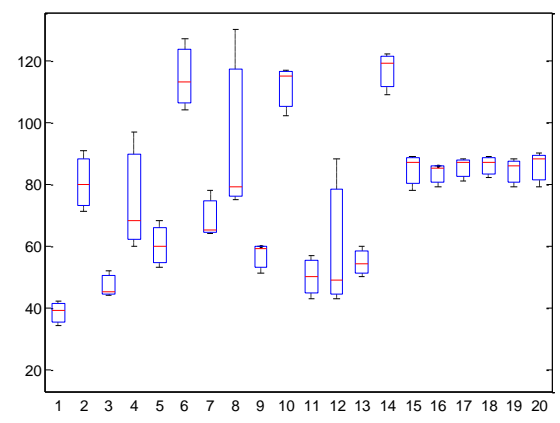

Figure 1. Box-plots of replicated response measures for each unit of Roman Catapult data.

By using the fuzzification rule, given in Equation (5), the data set with triangular fuzzy response values is obtained as in Table 2. 
The predicted fuzzy response model is obtained by using FLS approach as below:

$$
\begin{aligned}
\hat{\tilde{Y}}= & (75.1712,85.2183,92.5516)+(6.1973,14.6454,22.3317) X_{1} \\
& +(-7.9474,3.1401,12.4972) X_{2}+(11.1594,19.9862,27.1707) X_{3} \\
& +(-17.8744,-8.3820,-0.7558) X_{2}^{2}+(-15.75,-3.7625,6.75) X_{2} X_{3} .
\end{aligned}
$$

Table 2. The Roman Catapult data set with fuzzy responses

\begin{tabular}{cccccc}
\hline No & $\begin{array}{c}\text { Run } \\
\text { order }\end{array}$ & $X_{1}$ & $X_{2}$ & $X_{3}$ & $\tilde{\mathbf{Y}}$ \\
\hline 1 & 20 & -1 & -1 & -1 & $(34,38.944,42)$ \\
2 & 9 & -1 & -1 & 1 & $(71,83.36,91)$ \\
3 & 11 & -1 & 1 & -1 & $(44,48.944,52)$ \\
4 & 14 & -1 & 1 & 1 & $(60,82.866,97)$ \\
5 & 15 & 1 & -1 & -1 & $(53,62.27,68)$ \\
6 & 10 & 1 & -1 & 1 & $(104,118.214,127)$ \\
7 & 12 & 1 & 1 & -1 & $(64,72.652,78)$ \\
8 & 1 & 1 & 1 & 1 & $(75,108.99,130)$ \\
9 & 7 & -1.682 & 0 & 0 & $(51,56.562,60)$ \\
10 & 4 & 1.682 & 0 & 0 & $(102,111.27,117)$ \\
11 & 19 & 0 & -1.682 & 0 & $(43,51.652,57)$ \\
12 & 18 & 0 & 1.682 & 0 & $(43,70.81,88)$ \\
13 & 12 & 0 & 0 & -1.682 & $(50,56.18,60)$ \\
14 & 8 & 0 & 0 & 1.682 & $(109,117.034,122)$ \\
15 & 6 & 0 & 0 & 0 & $(78,84.798,89)$ \\
16 & 5 & 0 & 0 & 0 & $(79,83.326,86)$ \\
17 & 17 & 0 & 0 & 0 & $(81,85.326,88)$ \\
18 & 3 & 0 & 0 & 0 & $(82,86.326,89)$ \\
19 & 2 & 0 & 0 & 0 & $(79,84.562,88)$ \\
20 & 16 & 0 & 0 & 0 & $(79,85.798,90)$ \\
\hline
\end{tabular}

The predicted Bayesian model is taken into consideration as in the study of [11]. It is clear to say that the comparison of parameter estimates of fuzzy and Bayesian models is not possible unless transforming the parameter estimates to intervals. In this study, the same interval type Bayesian parameter estimates, which are obtained 95\% confident with HPD credible interval, are used as in the study of [11]. The previously obtained Bayesian interval estimates of model parameters with midpoint, width and radius metrics are summarized in Table 3.

Table 3. Interval type parameter estimates of Bayesian model with midpoint, width and radius metrics for Roman Catapult data set

\begin{tabular}{ccccc}
$\begin{array}{c}\text { Interval type } \\
\text { parameters } \\
{[\boldsymbol{\beta}]}\end{array}$ & $\left.\begin{array}{c}\text { Interval estimates } \\
\text { of parameters } \\
{[\boldsymbol{\beta}, \overline{\boldsymbol{\beta}}}\end{array}\right]$ & $\begin{array}{c}\text { Midpoint } \\
\operatorname{mid}(\boldsymbol{\beta})\end{array}$ & $\begin{array}{c}\text { Width } \\
w(\boldsymbol{\beta})\end{array}$ & $\begin{array}{c}\text { Radius } \\
\operatorname{Rad}(\boldsymbol{\beta})\end{array}$ \\
\hline$\left[\beta_{0}\right]$ & {$\left[\begin{array}{ll}77.3075 & 91.3816\end{array}\right]$} & 84.3445 & 14.0741 & 7.0371 \\
\hline$\left[\beta_{1}\right]$ & {$\left[\begin{array}{lllll}7.2240 & 20.5418\end{array}\right]$} & 13.8829 & 13.3178 & 6.6589 \\
\hline$\left[\beta_{2}\right]$ & {$\left[\begin{array}{lllll}-6.0375 & 7.2803\end{array}\right]$} & 0.6214 & 13.3178 & 6.6589 \\
\hline$\left[\beta_{3}\right]$ & {$\left[\begin{array}{lllll}11.9831 & 25.3009\end{array}\right]$} & 18.6420 & 13.3178 & 6.6589 \\
\hline$\left[\beta_{4}\right]$ & {$\left[\begin{array}{lllll}-17.2997 & -4.4539\end{array}\right]$} & -10.8768 & 12.8458 & 6.4229 \\
\hline$\left[\beta_{5}\right]$ & {$\left[\begin{array}{llll}-14.0757 & 3.3257\end{array}\right]$} & -5.3750 & 17.4014 & 8.7007 \\
\hline
\end{tabular}


In order to obtain interval type fuzzy parameter estimates, alpha-cut operation is applied to fuzzy parameter estimates. The predicted fuzzy model with alpha-cut level presentation of parameter estimates is given below

$$
\begin{aligned}
\hat{\tilde{Y}}= & {[75.1712+10.0471 \alpha, 92.5516-7.3333 \alpha]+[6.1973+8.4481 \alpha, 22.3317-7.6863 \alpha] X_{1} } \\
& +[-7.9474+11.0875 \alpha, 12.4972-9.3571 \alpha] X_{2}+[11.1594+8.8268 \alpha, 27.1707-7.1845 \alpha] X_{3} \\
& +[-17.8744+9.4925 \alpha,-0.7558-7.6262 \alpha] X_{2}^{2}+[-15.75+11.9875 \alpha, 6.75-10.5125 \alpha] X_{2} X_{3} .
\end{aligned}
$$

It is possible to obtain different real valued closed interval type parameter estimates by using different alpha-cut levels, $\alpha \in[0,1]$, e.g. $\alpha \in\{0,0.05,0.10, \ldots, 0.90,0.95\}$. The obtained interval type fuzzy parameter estimates, midpoint, width and radius metrics are presented in Table 4 for each alpha-cut levels.

\begin{tabular}{|c|c|c|c|c|c|}
\hline alpha-cut levels & $\begin{array}{c}\text { Interval type } \\
\text { parameters } \\
{\left[\boldsymbol{\beta}^{\alpha}\right]}\end{array}$ & $\begin{array}{c}\text { Interval estimates of } \\
\text { model parameters } \\
{\left[\underline{\boldsymbol{\beta}}^{\alpha}, \overline{\boldsymbol{\beta}}^{\alpha}\right]}\end{array}$ & $\begin{array}{l}\text { Midpoint } \\
\operatorname{mid}\left(\boldsymbol{\beta}^{\alpha}\right)\end{array}$ & $\begin{array}{l}\text { Width } \\
w\left(\boldsymbol{\beta}^{\alpha}\right)\end{array}$ & $\begin{array}{l}\text { Radius } \\
\operatorname{Rad}\left(\boldsymbol{\beta}^{\alpha}\right)\end{array}$ \\
\hline \multirow{6}{*}{$\mathbf{0}$} & {$\left[\beta_{0}^{\alpha}\right]$} & {$\left[\begin{array}{ll}75.1712 & 92.5516\end{array}\right]$} & 83.8614 & 17.3804 & 8.6902 \\
\hline & {$\left[\beta_{1}^{\alpha}\right]$} & {$\left[\begin{array}{ll}6.1973 & 22.3317\end{array}\right]$} & 14.2645 & 16.1344 & 8.0672 \\
\hline & {$\left[\beta_{2}^{\alpha}\right]$} & {$[-7.9474 \quad 12.4972]$} & 2.2749 & 20.4446 & 10.2223 \\
\hline & {$\left[\beta_{3}^{\alpha}\right]$} & {$\left[\begin{array}{ll}11.1594 & 27.1707\end{array}\right]$} & 19.165 & 16.0113 & 8.0056 \\
\hline & {$\left[\beta_{4}^{\alpha}\right.$} & {$\left[\begin{array}{ll}-17.8744 & -0.7558\end{array}\right]$} & -9.3151 & 17.1187 & 8.5593 \\
\hline & {$\left[\beta_{5}^{\alpha}\right]$} & {$[-15.75 \quad 6.75]$} & -4.5 & 22.5 & 11.25 \\
\hline \multirow{6}{*}{0.05} & $\beta_{0}^{\alpha}$ & {$\left[\begin{array}{ll}75.6736 & 92.1849]\end{array}\right.$} & 83.9292 & 16.5114 & 8.2557 \\
\hline & {$\left[\beta_{1}^{\alpha}\right]$} & {$\left[\begin{array}{ll}6.6197 & 21.94749\end{array}\right.$} & 14.2835 & 15.3277 & 7.6639 \\
\hline & $\beta_{2}^{\alpha}$ & {$\left[\begin{array}{ll}-7.3931 & 12.0294]\end{array}\right.$} & 2.3181 & 19.4224 & 9.7112 \\
\hline & $\beta_{3}^{\alpha}$ & {$[11.6008$ 26.8115] } & 19.2061 & 15.2107 & 7.6054 \\
\hline & $\beta_{4}^{\alpha}$ & {$\left[\begin{array}{ll}-17.3998 & -1.1371]\end{array}\right.$} & -9.2684 & 16.2627 & 8.1314 \\
\hline & {$\left[\beta_{5}^{\alpha}\right]$} & {$[-15.1506 \quad 6.2244]$} & -4.4631 & 21.3750 & 10.6875 \\
\hline \multirow{8}{*}{0.10} & {$\left[\beta_{0}^{\alpha}\right.$} & {$\left[\begin{array}{ll}76.1759 & 91.8183\end{array}\right]$} & 83.9971 & 15.6424 & 7.8212 \\
\hline & {$\left[\beta_{1}^{\alpha}\right]$} & {$\left[\begin{array}{ll}7.0421 & 21.5631\end{array}\right]$} & 14.3026 & 14.5210 & 7.2605 \\
\hline & {$\left[\beta_{2}^{\alpha}\right]$} & {$\left[\begin{array}{ll}-6.8387 & 11.5615]\end{array}\right.$} & 2.3614 & 18.4002 & 9.2001 \\
\hline & {$\left[\beta_{3}^{\alpha}\right]$} & {$\left[\begin{array}{ll}12.0421 & 26.4522\end{array}\right]$} & 19.2472 & 14.4101 & 7.2051 \\
\hline & {$\left[\beta_{4}^{\alpha}\right]$} & {$\left[\begin{array}{ll}-16.9252 & -1.5184\end{array}\right]$} & -9.2218 & 15.4068 & 7.7034 \\
\hline & {$\left[\beta_{5}^{\alpha}\right]$} & {$[-14.5513 \quad 5.6988]$} & -4.4262 & 20.2500 & 10.1250 \\
\hline & {$\left[\beta_{0}^{\alpha}\right]$} & {$\left[\begin{array}{ll}77.1806 & 91.0850\end{array}\right]$} & 84.1328 & 13.9043 & 6.9522 \\
\hline & {$\left[\beta_{1}^{\alpha}\right]$} & {$\left[\begin{array}{ll}7.8869 & 20.7945\end{array}\right]$} & 14.3407 & 12.9075 & 6.4538 \\
\hline
\end{tabular}

Table 4. Interval type parameter estimates of fuzzy model with midpoint, width and radius metrics for Roman Catapult data set 


\begin{tabular}{|c|c|c|c|c|c|}
\hline \multirow{4}{*}{0.20} & {$\left[\beta_{2}^{\alpha}\right]$} & {$[-5.7299 \quad 10.6258]$} & 2.4479 & 16.3557 & 8.1779 \\
\hline & {$\left[\beta_{3}^{\alpha}\right]$} & {$\left[\begin{array}{ll}12.9248 & 25.7338\end{array}\right]$} & 19.3293 & 12.8090 & 6.4045 \\
\hline & {$\left[\beta_{4}^{\alpha}\right]$} & {$\left[\begin{array}{ll}-15.9759 & -2.281\end{array}\right]$} & -9.1285 & 13.6949 & 6.8475 \\
\hline & {$\left[\beta_{5}^{\alpha}\right]$} & {$[-13.3525 \quad 4.6475]$} & -4.3525 & 18.0000 & 9.0000 \\
\hline \multirow{6}{*}{0.30} & {$\left[\beta_{0}^{\alpha}\right]$} & {$\left[\begin{array}{ll}78.1853 & 90.3516\end{array}\right]$} & 84.2685 & 12.1663 & 6.0831 \\
\hline & {$\left[\beta_{1}^{\alpha}\right]$} & {$\left[\begin{array}{ll}8.7317 & 20.0258\end{array}\right]$} & 14.3788 & 11.2941 & 5.6470 \\
\hline & {$\left[\beta_{2}^{\alpha}\right]$} & {$\left[\begin{array}{ll}-4.6212 & 9.6901\end{array}\right]$} & 2.5344 & 14.3112 & 7.1556 \\
\hline & {$\left[\beta_{3}^{\alpha}\right]$} & {$\left[\begin{array}{ll}13.8074 & 25.0153\end{array}\right]$} & 19.4114 & 11.2079 & 5.6039 \\
\hline & {$\left[\beta_{4}^{\alpha}\right]$} & {$\left[\begin{array}{ll}-15.0267 & -3.0436\end{array}\right]$} & -9.0352 & 11.9831 & 5.9915 \\
\hline & {$\left[\beta_{5}^{\alpha}\right]$} & {$\left[\begin{array}{ll}-12.1538 & 3.5963]\end{array}\right.$} & -4.2788 & 15.75 & 7.875 \\
\hline \multirow{6}{*}{0.40} & {$\left[\beta_{0}^{\alpha}\right]$} & {$\left[\begin{array}{ll}79.1901 & 89.6183]\end{array}\right.$} & 84.4042 & 10.4282 & 5.2141 \\
\hline & {$\left[\beta_{1}^{\alpha}\right]$} & {$\left[\begin{array}{ll}9.5765 & 19.2572\end{array}\right]$} & 14.4169 & 9.6807 & 4.8403 \\
\hline & {$\left[\beta_{2}^{\alpha}\right]$} & {$[-3.5124 \quad 8.7544]$} & 2.621 & 12.2668 & 6.1334 \\
\hline & {$\left[\beta_{3}^{\alpha}\right]$} & {$\left[\begin{array}{ll}14.6901 & 24.2969\end{array}\right]$} & 19.4935 & 9.6068 & 4.8034 \\
\hline & {$\left[\beta_{4}^{\alpha}\right]$} & {$\left[\begin{array}{ll}-14.0774 & -3.8062\end{array}\right]$} & -8.9418 & 10.2712 & 5.1356 \\
\hline & {$\left[\beta_{5}^{\alpha}\right]$} & {$[-10.9550 \quad 2.545]$} & -4.205 & 13.5 & 6.75 \\
\hline \multirow{6}{*}{0.50} & {$\left[\beta_{0}^{\alpha}\right]$} & {$\left[\begin{array}{ll}80.1948 & 88.885\end{array}\right]$} & 84.5399 & 8.6902 & 4.3451 \\
\hline & $\left.\beta_{1}^{\alpha}\right]$ & {$\left[\begin{array}{ll}10.4214 & 18.4886\end{array}\right]$} & 14.4550 & 8.0672 & 4.0336 \\
\hline & {$\left[\beta_{2}^{\alpha}\right]$} & {$\left[\begin{array}{ll}-2.4037 & 7.8186\end{array}\right]$} & 2.7075 & 10.2223 & 5.1112 \\
\hline & {$\left[\beta_{3}^{\alpha}\right]$} & {$\left[\begin{array}{ll}15.5728 & 23.5784\end{array}\right]$} & 19.5756 & 8.0056 & 4.0028 \\
\hline & {$\left[\beta_{4}^{\alpha}\right]$} & {$\left[\begin{array}{ll}-13.1282 & -4.5689\end{array}\right]$} & -8.8485 & 8.5593 & 4.2797 \\
\hline & {$\left[\beta_{5}^{\alpha}\right]$} & {$\left[\begin{array}{ll}-9.7562 & 1.4938\end{array}\right]$} & -4.1312 & 11.25 & 5.625 \\
\hline \multirow{9}{*}{0.60} & {$\left[\beta_{0}^{\alpha}\right]$} & {$[81.1995 \quad 88.1516]$} & 84.6756 & 6.9522 & 3.4761 \\
\hline & {$\left[\beta_{1}^{\alpha}\right]$} & [11.2662 17.7199] & 14.4931 & 6.4538 & 3.2269 \\
\hline & {$\left[\beta_{2}^{\alpha}\right]$} & {$[-1.2949 \quad 6.8829]$} & 2.7940 & 8.1779 & 4.0889 \\
\hline & {$\left[\beta_{3}^{\alpha}\right]$} & {$\left[\begin{array}{ll}16.4555 & 22.86\end{array}\right]$} & 19.6577 & 6.4045 & 3.2023 \\
\hline & {$\left[\beta_{4}^{\alpha}\right]$} & {$\left[\begin{array}{ll}-12.1790 & -5.3315\end{array}\right]$} & -8.7552 & 6.8475 & 3.4237 \\
\hline & {$\left[\beta_{5}^{\alpha}\right]$} & {$[-8.5575 \quad 0.4425]$} & -4.0575 & 9 & 4.5 \\
\hline & {$\left[\beta_{0}^{\alpha}\right]$} & {$[82.2042 \quad 87.4183]$} & 84.8113 & 5.2141 & 2.6071 \\
\hline & {$\left[\beta_{1}^{\alpha}\right]$} & {$\left[\begin{array}{ll}12.1110 & 16.9513]\end{array}\right.$} & 14.5311 & 4.8403 & 2.4202 \\
\hline & {$\left[\beta_{2}^{\alpha}\right]$} & {$[-0.1862$} & 2.8805 & 6.1334 & 3.0667 \\
\hline
\end{tabular}




\begin{tabular}{|c|c|c|c|c|c|c|}
\hline \multirow[t]{3}{*}{0.70} & {$\left[\beta_{3}^{\alpha}\right]$} & {$[17.3382$} & 22.1415] & 19.7399 & 4.8034 & 2.4017 \\
\hline & {$\left[\beta_{4}^{\alpha}\right]$} & {$[-11.2297$} & $-6.0941]$ & -8.6619 & 5.1356 & 2.5678 \\
\hline & {$\left[\beta_{5}^{\alpha}\right]$} & {$[-7.3588$} & $-0.6087]$ & -3.9837 & 6.75 & 3.375 \\
\hline \multirow{6}{*}{0.80} & {$\left[\beta_{0}^{\alpha}\right]$} & [83.2089 & 86.685] & 84.947 & 3.4761 & 1.738 \\
\hline & {$\left[\beta_{1}^{\alpha}\right]$} & {$[12.9558$} & 16.1827] & 14.5692 & 3.2269 & 1.6134 \\
\hline & {$\left[\beta_{2}^{\alpha}\right]$} & {$[0.9226$} & 5.0115] & 2.9670 & 4.0889 & 2.0445 \\
\hline & {$\left[\beta_{3}^{\alpha}\right]$} & {$[18.2208$} & 21.4231] & 19.8220 & 3.2023 & 1.6011 \\
\hline & {$\left[\beta_{4}^{\alpha}\right]$} & {$[-10.2805$} & $-6.8567]$ & -8.5686 & 3.4237 & 1.7119 \\
\hline & {$\left[\beta_{5}^{\alpha}\right]$} & {$[-6.1600$} & $-1.66]$ & -3.9100 & 4.5 & 2.25 \\
\hline \multirow{6}{*}{0.90} & {$\left[\beta_{0}^{\alpha}\right]$} & {$[84.2136$} & 85.9517] & 85.0826 & 1.738 & 0.869 \\
\hline & {$\left[\beta_{1}^{\alpha}\right]$} & {$[13.8006$} & $15.4141]$ & 14.6073 & 1.6134 & 0.8067 \\
\hline & {$\left[\beta_{2}^{\alpha}\right]$} & {$[2.0313$} & $4.0758]$ & 3.0535 & 2.0445 & 1.0222 \\
\hline & {$\left[\beta_{3}^{\alpha}\right]$} & [19.1035 & $20.7046]$ & 19.9041 & 1.6011 & 0.8006 \\
\hline & {$\left[\beta_{4}^{\alpha}\right]$} & {$[-9.3312$} & $-7.6193]$ & -8.4753 & 1.7119 & 0.8559 \\
\hline & {$\left[\beta_{5}^{\alpha}\right]$} & {$[-4.9612$} & $-2.7112]$ & -3.8362 & 2.25 & 1.125 \\
\hline \multirow{6}{*}{0.95} & {$\left[\beta_{0}^{\alpha}\right]$} & {$[84.716$} & $85.585]$ & 85.1505 & 0.869 & 0.4345 \\
\hline & {$\left[\beta_{1}^{\alpha}\right]$} & {$[14.2230$} & 15.0297] & 14.6264 & 0.8067 & 0.4034 \\
\hline & {$\left[\beta_{2}^{\alpha}\right]$} & {$[2.5857$} & $3.6079]$ & 3.0968 & 1.0222 & 0.5111 \\
\hline & {$\left[\beta_{3}^{\alpha}\right]$} & [19.5449 & 20.3454] & 19.9451 & 0.8006 & 0.4003 \\
\hline & {$\left[\beta_{4}^{\alpha}\right]$} & {$[-8.8566$} & $-8.0006]$ & -8.4286 & 0.8559 & 0.428 \\
\hline & {$\left[\beta_{5}^{\alpha}\right]$} & {$[-4.3619$} & $-3.2369]$ & -3.7994 & 1.125 & 0.5625 \\
\hline
\end{tabular}

It can be easily seen from Table 4 that the larger the alpha-cut levels, the smaller the $w$ metric values. The small value of $w$ metric means that the variation of parameter estimates is small. In order to define the similarities between interval type parameter estimates of Bayesian model, given in Table 3, and interval type parameter estimates of fuzzy model, given in Table 4, Hausdorff metric is calculated. The obtained results are presented in Table 5.

Table 5. Hausdorff metric of interval type parameter estimates for Roman Catapult data set

\begin{tabular}{cccccccc}
\hline $\begin{array}{c}\text { alpha-cut } \\
\text { levels }\end{array}$ & $H_{1}$ & $H_{2}$ & $H_{3}$ & $H_{4}$ & $H_{5}$ & $H_{6}$ & $H_{7}$ \\
\hline $\mathbf{0}$ & 2.1363 & 1.7899 & 5.2169 & 1.8698 & 3.6981 & 3.4243 & 18.1353 \\
$\mathbf{0 . 0 5}$ & 1.6339 & 1.4056 & 4.7491 & 1.5106 & 3.3168 & 2.8987 & 15.5147 \\
$\mathbf{0 . 1 0}$ & 1.1316 & 1.0213 & 4.2812 & 1.1513 & 2.9355 & 2.3731 & 12.8940 \\
$\mathbf{0 . 2 0}$ & $\mathbf{0 . 2 9 6 6}$ & $\mathbf{0 . 6 6 2 9}$ & 3.3455 & $\mathbf{0 . 9 4 1 7}$ & $\mathbf{2 . 1 7 2 9}$ & $\mathbf{1 . 3 2 1 8}$ & $\mathbf{8 . 7 4 1 4}$ \\
$\mathbf{0 . 3 0}$ & 1.0300 & 1.5077 & $\mathbf{2 . 4 0 9 8}$ & 1.8243 & 2.2730 & 1.9219 & 10.9668 \\
$\mathbf{0 . 4 0}$ & 1.8826 & 2.3525 & 2.5251 & 2.7070 & 3.2223 & 3.1207 & 15.8101 \\
$\mathbf{0 . 5 0}$ & 2.8873 & 3.1974 & 3.6338 & 3.5897 & 4.1715 & 4.3194 & 21.7991 \\
$\mathbf{0 . 6 0}$ & 3.8920 & 4.0422 & 4.7426 & 4.4724 & 5.1207 & 5.5182 & 27.7880 \\
\hline
\end{tabular}




\begin{tabular}{llllllll}
\hline $\mathbf{0 . 7 0}$ & 4.8967 & 4.8870 & 5.8513 & 5.3551 & 6.0700 & 6.7169 & 33.7770 \\
$\mathbf{0 . 8 0}$ & 5.9014 & 5.7318 & 6.9601 & 6.2377 & 7.0192 & 7.9157 & 39.7660 \\
$\mathbf{0 . 9 0}$ & 6.9061 & 6.5766 & 8.0688 & 7.1204 & 7.9685 & 9.1144 & 45.7549 \\
$\mathbf{0 . 9 5}$ & 7.4085 & 6.9990 & 8.6232 & 7.5618 & 8.4431 & 9.7138 & 48.7494 \\
\hline
\end{tabular}

From Table 5, it is possible to say that the small $H$ values are obtained for alpha-cut level is between 0.20 and 0.30 roughly. The smaller the $H$ values, the higher the similarity of interval type parameter estimates. According to the $H$ metric values in Table 5, someone can prefer to use fuzzy modeling approach for $\alpha \in[0.20,0.30]$ - cut level instead of using Bayesian approach for $95 \%$ confident without making any assumptions for interval type parameter estimates.

Printing Ink Data Set: Printing Ink data set is originally used in the studies of [30] to model printing machine ability. The printing ink process is designed with three inputs (speed $-X_{1}$, pressure $-X_{2}$, distance $-X_{3}$ ) and three replicated response measures by using $3^{3}$ full factorial design. In this study, the same interval type Bayesian parameter estimates, which are obtained 95\% confident with HPD credible interval, are used as in the study of [11]. The previously obtained Bayesian interval estimates of model parameters with midpoint, width and radius metrics are summarized in Table 6.

Table 6. Interval type parameter estimates of Bayesian model with midpoint, width and radius metrics for Printing Ink data set

\begin{tabular}{ccccc}
\hline $\begin{array}{c}\text { Interval } \\
\text { type } \\
\text { parameters } \\
{[\boldsymbol{\beta}]}\end{array}$ & $\begin{array}{c}\text { Interval estimates } \\
\text { of parameters } \\
{[\boldsymbol{\beta}, \overline{\boldsymbol{\beta}}]}\end{array}$ & $\begin{array}{c}\text { Midpoint } \\
\operatorname{mid}(\boldsymbol{\beta})\end{array}$ & $\begin{array}{c}\text { Width } \\
w(\boldsymbol{\beta})\end{array}$ & $\begin{array}{c}\text { Radius } \\
\operatorname{Rad}(\boldsymbol{\beta})\end{array}$ \\
\hline$\left[\beta_{0}\right]$ & {$\left[\begin{array}{lllll}274.8511 & 354.4823\end{array}\right]$} & 314.6667 & 79.6312 & 39.8156 \\
\hline$\left[\beta_{1}\right]$ & {$\left[\begin{array}{lllll}128.236 & 225.764\end{array}\right]$} & 177 & 97.528 & 48.764 \\
\hline$\left[\beta_{2}\right]$ & {$\left[\begin{array}{llll}60.662 & 158.1899\end{array}\right]$} & 109.426 & 97.5279 & 48.7639 \\
\hline$\left[\beta_{3}\right]$ & {$\left[\begin{array}{llll}82.699 & 180.2269\end{array}\right]$} & 131.463 & 97.5279 & 48.764 \\
\hline$\left[\beta_{4}\right]$ & {$\left[\begin{array}{llll}6.3044 & 125.7512\end{array}\right]$} & 66.0278 & 119.4468 & 59.7234 \\
\hline$\left[\beta_{5}\right]$ & {$\left[\begin{array}{llll}15.7488 & 135.1956\end{array}\right]$} & 75.4722 & 119.4468 & 59.7234 \\
\hline$\left[\beta_{6}\right]$ & {$\left[\begin{array}{llll}-16.1401 & 103.3067\end{array}\right]$} & 43.5833 & 119.4468 & 59.7234 \\
\hline
\end{tabular}

In order to understand the data structure of the replicated measures, box-plots of the responses were plotted. In this study, fuzzification rule, given in Equation (5), is used for replicated response measures since the replicated measures have skewed distribution, presented with box-plots in Figure 2 for each unit. 


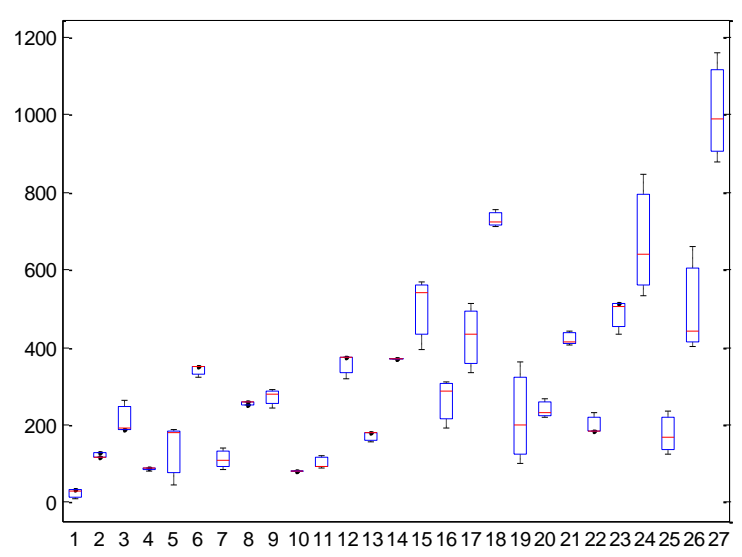

Figure 2. Box-plots of replicated response measures for each unit of Printing Ink data set.

The printing ink data set with fuzzy response values is given in Table 7.

Table 7. The Printing Ink data set with fuzzy responses

\begin{tabular}{ccccc}
\hline No & $X_{1}$ & $X_{2}$ & $X_{3}$ & $\tilde{\mathbf{Y}}$ \\
\hline $\mathbf{1}$ & -1 & -1 & -1 & $(10,24.8,34)$ \\
$\mathbf{2}$ & 0 & -1 & -1 & $(115,124.3,130)$ \\
$\mathbf{3}$ & 1 & -1 & -1 & $(186,233.6,263)$ \\
$\mathbf{4}$ & -1 & 0 & -1 & $(82,85.7,88)$ \\
$\mathbf{5}$ & 0 & 0 & -1 & $(44,133,188)$ \\
$\mathbf{6}$ & 1 & 0 & -1 & $(322,339.3,350)$ \\
$\mathbf{7}$ & -1 & 1 & -1 & $(86,120,141)$ \\
$\mathbf{8}$ & 0 & 1 & -1 & $(251,255.9,259)$ \\
$\mathbf{9}$ & 1 & 1 & -1 & $(245,272.8,290)$ \\
$\mathbf{1 0}$ & -1 & -1 & 0 & $(81,81,81)$ \\
$\mathbf{1 1}$ & 0 & -1 & 0 & $(90,109.8,122)$ \\
$\mathbf{1 2}$ & 1 & -1 & 0 & $(319,354.2,376)$ \\
$\mathbf{1 3}$ & -1 & 0 & 0 & $(154,170.1,180)$ \\
$\mathbf{1 4}$ & 0 & 0 & 0 & $(372,372,372)$ \\
$\mathbf{1 5}$ & 1 & 0 & 0 & $(396,502.3,568)$ \\
$\mathbf{1 6}$ & -1 & 1 & 0 & $(192,266.2,312)$ \\
$\mathbf{1 7}$ & 0 & 1 & 0 & $(336,445.4,513)$ \\
$\mathbf{1 8}$ & 1 & 1 & 0 & $(713,738.3,754)$ \\
$\mathbf{1 9}$ & -1 & -1 & 1 & $(99,262.8,364)$ \\
$\mathbf{2 0}$ & 0 & -1 & 1 & $(221,248.8,266)$ \\
$\mathbf{2 1}$ & 1 & -1 & 1 & $(408,429.6,443)$ \\
$\mathbf{2 2}$ & -1 & 0 & 1 & $(182,213.5,233)$ \\
$\mathbf{2 3}$ & 0 & 0 & 1 & $(434,484.1,515)$ \\
$\mathbf{2 4}$ & 1 & 0 & 1 & $(535,727.2,846)$ \\
$\mathbf{2 5}$ & -1 & 1 & 1 & $(126,194,236)$ \\
$\mathbf{2 6}$ & 0 & 1 & 1 & $(403,561.8,660)$ \\
$\mathbf{2 7}$ & 1 & 1 & 1 & $(878,1052.9,1161)$ \\
\hline & & & & \\
\end{tabular}

The predicted fuzzy response model, with alpha-cut parameter estimates, is obtained as 


$$
\begin{aligned}
\hat{\tilde{Y}} & =[269.6296+56.4211 \alpha, 360.9259-34.8752 \alpha]+[129.6111+49.9587 \alpha, 224.3889-44.8191 \alpha] X_{1} \\
& +[63.9444+49.3016 \alpha, 155.3889-42.1429 \alpha] X_{2}+[85.7222+57.9027 \alpha, 187.9444-44.3196 \alpha] X_{3} \\
& +[21.25+48.3393 \alpha, 113.9167-44.3273 \alpha] X_{1} X_{2}+[21.9167+55.107 \alpha, 129.4167-52.393 \alpha] X_{1} X_{3} \\
& +[-3.75+53.8695 \alpha, 97.8333-47.7138 \alpha] X_{2} X_{3} .
\end{aligned}
$$

The obtained interval type fuzzy parameter estimates, midpoint, width and radius metrics are presented in

\begin{tabular}{|c|c|c|c|c|c|}
\hline alpha-cut levels & $\begin{array}{c}\text { Interval type } \\
\text { parameters } \\
{\left[\boldsymbol{\beta}^{\alpha}\right]}\end{array}$ & 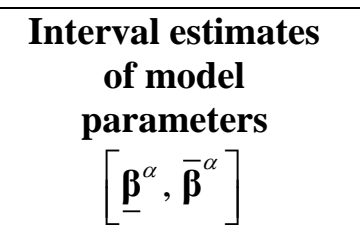 & $\begin{array}{l}\text { Midpoint } \\
\operatorname{mid}\left(\boldsymbol{\beta}^{\alpha}\right)\end{array}$ & $\begin{array}{l}\text { Width } \\
w\left(\boldsymbol{\beta}^{\alpha}\right)\end{array}$ & $\begin{array}{c}\text { Radius } \\
\operatorname{Rad}\left(\boldsymbol{\beta}^{\alpha}\right)\end{array}$ \\
\hline \multirow{7}{*}{$\mathbf{0}$} & {$\left[\beta_{0}^{\alpha}\right]$} & [269.6296 360.9259$]$ & 315.2778 & 91.2963 & 45.6481 \\
\hline & {$\left[\beta_{1}^{\alpha}\right]$} & [129.6111 224.3889] & 177.0000 & 94.7778 & 47.3889 \\
\hline & {$\left[\beta_{2}^{\alpha}\right]$} & {$\left[\begin{array}{ll}63.9444 & 155.3889\end{array}\right]$} & 109.6667 & 91.4444 & 45.7222 \\
\hline & {$\left[\beta_{3}^{\alpha}\right]$} & {$\left[\begin{array}{lll}85.7222 & 187.9444\end{array}\right]$} & 136.8333 & $\begin{array}{c}102.222 \\
2 \\
\end{array}$ & 51.1111 \\
\hline & $\beta_{4}^{\alpha}$ & [21.25 113.9167] & 67.5833 & 92.6667 & 46.3333 \\
\hline & {$\left[\beta_{5}^{\alpha}\right]$} & [21.9167 129.4167] & 75.6667 & $\begin{array}{c}107.500 \\
0\end{array}$ & 53.7500 \\
\hline & {$\left[\beta_{6}^{\alpha}\right]$} & {$\left[\begin{array}{ll}-3.75 & 97.8333\end{array}\right]$} & 47.0417 & $\begin{array}{c}101.583 \\
3 \\
\end{array}$ & 50.7917 \\
\hline \multirow{7}{*}{0.05} & {$\left[\beta_{0}^{\alpha}\right]$} & {$\left[\begin{array}{lll}272.4507 & 359.1822\end{array}\right]$} & 315.8164 & 86.7315 & 43.3657 \\
\hline & {$\left[\beta_{1}^{\alpha}\right]$} & {$\left[\begin{array}{ll}132.109 & 222.1479\end{array}\right]$} & 177.1285 & 90.0389 & 45.0194 \\
\hline & {$\left[\beta_{2}^{\alpha}\right]$} & {$\left[\begin{array}{ll}66.4095 & 153.2817\end{array}\right]$} & 109.8456 & 86.8722 & 43.4361 \\
\hline & {$\left[\beta_{3}^{\alpha}\right]$} & {$\left[\begin{array}{ll}88.6174 & 185.7285\end{array}\right]$} & 137.1729 & 97.1111 & 48.5556 \\
\hline & {$\left[\beta_{4}^{\alpha}\right]$} & {$\left[\begin{array}{lll}23.667 & 111.7003\end{array}\right]$} & 67.6836 & 88.0333 & 44.0167 \\
\hline & {$\left[\beta_{5}^{\alpha}\right]$} & [24.672 126.797$]$ & 75.7345 & $\begin{array}{c}102.125 \\
0 \\
\end{array}$ & 51.0625 \\
\hline & {$\left[\beta_{6}^{\alpha}\right]$} & {$\left[\begin{array}{ll}-1.0565 & 95.4476\end{array}\right]$} & 47.1956 & 96.5042 & 48.2521 \\
\hline \multirow{7}{*}{0.10} & {$\left[\beta_{0}^{\alpha}\right]$} & {$\left[\begin{array}{ll}275.2717 & 357.4384\end{array}\right]$} & 316.3551 & 82.1667 & 41.0833 \\
\hline & {$\left[\beta_{1}^{\alpha}\right]$} & [134.607 219.907] & 177.2570 & 85.3000 & 42.6500 \\
\hline & {$\left[\beta_{2}^{\alpha}\right]$} & {$\left[\begin{array}{ll}68.8746 & 151.1746\end{array}\right]$} & 110.0246 & 82.3000 & 41.1500 \\
\hline & {$\left[\beta_{3}^{\alpha}\right]$} & 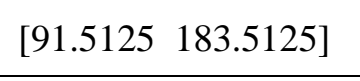 & 137.5125 & 92.0000 & 46.0000 \\
\hline & {$\left[\beta_{4}^{\alpha}\right]$} & [26.0839 109.4839$]$ & 67.7839 & 83.4000 & 41.7000 \\
\hline & {$\left[\beta_{5}^{\alpha}\right]$} & {$\left[\begin{array}{ll}27.4274 & 124.1774\end{array}\right]$} & 75.8024 & 96.7500 & 48.3750 \\
\hline & {$\left[\beta_{6}^{\alpha}\right]$} & {$\left[\begin{array}{ll}1.6369 & 93.062\end{array}\right]$} & 47.3494 & 91.4250 & 45.7125 \\
\hline
\end{tabular}
Table 8 for each alpha-cut levels.

Table 8. Interval type parameter estimates of fuzzy model with midpoint, width and radius metrics for Printing Ink data set 


\begin{tabular}{|c|c|c|c|c|c|}
\hline \multirow{7}{*}{0.20} & {$\left[\beta_{0}^{\alpha}\right]$} & [280.9139 353.9509$]$ & 317.4324 & 73.0370 & 36.5185 \\
\hline & {$\left[\beta_{1}^{\alpha}\right]$} & {$\left[\begin{array}{ll}139.6028 & 215.4251\end{array}\right]$} & 177.5140 & 75.8222 & 37.9111 \\
\hline & {$\left[\beta_{2}^{\alpha}\right]$} & {$\left[\begin{array}{ll}73.8048 & 146.9603\end{array}\right]$} & 110.3825 & 73.1556 & 36.5778 \\
\hline & {$\left[\beta_{3}^{\alpha}\right]$} & {$\left[\begin{array}{ll}97.3028 & 179.0805\end{array}\right]$} & 138.1916 & 81.7778 & 40.8889 \\
\hline & {$\left[\beta_{4}^{\alpha}\right]$} & {$\left[\begin{array}{ll}30.9179 & 105.0512\end{array}\right]$} & 67.9845 & 74.1333 & 37.0667 \\
\hline & {$\left[\beta_{5}^{\alpha}\right]$} & {$\left[\begin{array}{ll}32.9381 & 118.9381\end{array}\right]$} & 75.9381 & 86.0000 & 43.0000 \\
\hline & $\left.\beta_{6}^{\alpha}\right]$ & {$\left[\begin{array}{ll}7.0239 & 88.2906\end{array}\right]$} & 47.6572 & 81.2667 & 40.6333 \\
\hline \multirow{7}{*}{0.30} & {$\left[\beta_{0}^{\alpha}\right]$} & {$\left[\begin{array}{lll}286.556 & 350.4634\end{array}\right]$} & 318.5097 & 63.9074 & 31.9537 \\
\hline & $\left.\beta_{1}^{\alpha}\right]$ & [144.5987 210.9432] & 177.7709 & 66.3444 & 33.1722 \\
\hline & {$\left[\beta_{2}^{\alpha}\right]$} & {$\left[\begin{array}{lll}78.7349 & 142.746\end{array}\right]$} & 110.7405 & 64.0111 & 32.0056 \\
\hline & {$\left[\beta_{3}^{\alpha}\right]$} & [103.093 174.6486$]$ & 138.8708 & 71.5556 & 35.7778 \\
\hline & {$\left[\beta_{4}^{\alpha}\right]$} & {$\left[\begin{array}{ll}35.7518 & 100.6185\end{array}\right]$} & 68.1851 & 64.8667 & 32.4333 \\
\hline & {$\left[\beta_{5}^{\alpha}\right]$} & {$\left[\begin{array}{ll}38.4488 & 113.6988\end{array}\right]$} & 76.0738 & 75.2500 & 37.6250 \\
\hline & {$\left[\beta_{6}^{\alpha}\right]$} & {$\left[\begin{array}{ll}12.4108 & 83.5192\end{array}\right]$} & 47.9650 & 71.1083 & 35.5542 \\
\hline \multirow{7}{*}{0.40} & {$\left[\beta_{0}^{\alpha}\right]$} & [292.1981 346.9759] & 319.5870 & 54.7778 & 27.3889 \\
\hline & {$\left[\beta_{1}^{\alpha}\right]$} & [149.5946 206.4612] & 178.0279 & 56.8667 & 28.4333 \\
\hline & $\left.\beta_{2}^{\alpha}\right]$ & {$\left[\begin{array}{ll}83.6651 & 138.5317\end{array}\right]$} & 111.0984 & 54.8667 & 27.4333 \\
\hline & {$\left[\beta_{3}^{\alpha}\right]$} & [108.8833 170.2166$]$ & 139.5500 & 61.3333 & 30.6667 \\
\hline & {$\left[\beta_{4}^{\alpha}\right]$} & {$\left[\begin{array}{ll}40.5857 & 96.1857\end{array}\right]$} & 68.3857 & 55.6000 & 27.8000 \\
\hline & {$\left[\beta_{5}^{\alpha}\right]$} & {$\left[\begin{array}{ll}43.9595 & 108.4595\end{array}\right]$} & 76.2095 & 64.5000 & 32.2500 \\
\hline & {$\left[\beta_{6}^{\alpha}\right]$} & {$\left[\begin{array}{ll}17.7978 & 78.7478\end{array}\right]$} & 48.2728 & 60.9500 & 30.4750 \\
\hline \multirow{7}{*}{0.50} & {$\left[\beta_{0}^{\alpha}\right]$} & [297.8402 343.4883] & 320.6643 & 45.6481 & 22.8241 \\
\hline & {$\left[\beta_{1}^{\alpha}\right]$} & [154.5904 201.9793] & 178.2849 & 47.3889 & 23.6944 \\
\hline & {$\left[\beta_{2}^{\alpha}\right]$} & {$\left[\begin{array}{ll}88.5952 & 134.3174\end{array}\right]$} & 111.4563 & 45.7222 & 22.8611 \\
\hline & {$\left[\beta_{3}^{\alpha}\right]$} & {$\left[\begin{array}{ll}114.6736 & 165.7847]\end{array}\right.$} & 140.2291 & 51.1111 & 25.5556 \\
\hline & {$\left[\beta_{4}^{\alpha}\right]$} & {$\left[\begin{array}{ll}45.4197 & 91.753\end{array}\right]$} & 68.5863 & 46.3333 & 23.1667 \\
\hline & {$\left[\beta_{5}^{\alpha}\right]$} & {$\left[\begin{array}{lll}49.4702 & 103.2202\end{array}\right]$} & 76.3452 & 53.7500 & 26.8750 \\
\hline & {$\left[\beta_{6}^{\alpha}\right]$} & {$[23.1847 \quad 73.9764]$} & 48.5806 & 50.7917 & 25.3958 \\
\hline \multirow[b]{3}{*}{0.60} & {$\left[\beta_{0}^{\alpha}\right]$} & {$\left[\begin{array}{ll}303.4823 & 340.0008\end{array}\right]$} & 321.7416 & 36.5185 & 18.2593 \\
\hline & {$\left[\beta_{1}^{\alpha}\right]$} & [159.5863 197.4974] & 178.5419 & 37.9111 & 18.9556 \\
\hline & {$\left[\beta_{2}^{\alpha}\right]$} & {$\left[\begin{array}{lll}93.5254 & 130.1032\end{array}\right]$} & 111.8143 & 36.5778 & 18.2889 \\
\hline
\end{tabular}




\begin{tabular}{|c|c|c|c|c|c|c|}
\hline & {$\left[\beta_{3}^{\alpha}\right]$} & {$[120.4638$} & 161.3527] & 140.9083 & 40.8889 & 20.4444 \\
\hline & {$\left[\beta_{4}^{\alpha}\right]$} & {$[50.2536$} & 87.3203] & 68.7869 & 37.0667 & 18.5333 \\
\hline & {$\left[\beta_{5}^{\alpha}\right]$} & {$[54.9809$} & 97.9809] & 76.4809 & 43.0000 & 21.5000 \\
\hline & {$\left[\beta_{6}^{\alpha}\right]$} & {$[28.5717$} & 69.205] & 48.8884 & 40.6333 & 20.3167 \\
\hline \multirow{7}{*}{0.70} & {$\left[\beta_{0}^{\alpha}\right]$} & {$[309.1244$} & 336.5133] & 322.8189 & 27.3889 & 13.6944 \\
\hline & {$\left[\beta_{1}^{\alpha}\right]$} & {$[164.5822$} & $193.0155]$ & 178.7988 & 28.4333 & 14.2167 \\
\hline & {$\left[\beta_{2}^{\alpha}\right]$} & {$[98.4555$} & 125.8889] & 112.1722 & 27.4333 & 13.7167 \\
\hline & {$\left[\beta_{3}^{\alpha}\right]$} & {$[126.2541$} & $156.9208]$ & 141.5874 & 30.6667 & 15.3333 \\
\hline & {$\left[\beta_{4}^{\alpha}\right]$} & {$[55.0875$} & 82.8875] & 68.9875 & 27.8000 & 13.9000 \\
\hline & {$\left[\beta_{5}^{\alpha}\right]$} & {$[60.4916$} & 92.7416] & 76.6166 & 32.2500 & 16.1250 \\
\hline & {$\left[\beta_{6}^{\alpha}\right]$} & [33.9586 & 64.4337] & 49.1961 & 30.4750 & 15.2375 \\
\hline \multirow{7}{*}{0.80} & {$\left[\beta_{0}^{\alpha}\right]$} & {$[314.7665$} & $333.0258]$ & 323.8961 & 18.2593 & 9.1296 \\
\hline & {$\left[\beta_{1}^{\alpha}\right]$} & {$[169.578$} & 188.5336] & 179.0558 & 18.9556 & 9.4778 \\
\hline & {$\left[\beta_{2}^{\alpha}\right]$} & {$[103.3857$} & 121.6746] & 112.5301 & 18.2889 & 9.1444 \\
\hline & {$\left[\beta_{3}^{\alpha}\right]$} & {$[132.0444$} & $152.4888]$ & 142.2666 & 20.4444 & 10.2222 \\
\hline & {$\left[\beta_{4}^{\alpha}\right]$} & [59.9215 & 78.4548] & 69.1881 & 18.5333 & 9.2667 \\
\hline & {$\left[\beta_{5}^{\alpha}\right]$} & {$[66.0023$} & 87.5023] & 76.7523 & 21.5000 & 10.7500 \\
\hline & {$\left[\beta_{6}^{\alpha}\right]$} & {$[39.3456$} & 59.6623] & 49.5039 & 20.3167 & 10.1583 \\
\hline \multirow{7}{*}{0.90} & {$\left[\beta_{0}^{\alpha}\right]$} & {$[320.4086$} & 329.5383] & 324.9734 & 9.1296 & 4.5648 \\
\hline & {$\left[\beta_{1}^{\alpha}\right]$} & {$[174.5739$} & 184.0517] & 179.3128 & 9.4778 & 4.7389 \\
\hline & {$\left[\beta_{2}^{\alpha}\right]$} & {$[108.3158$} & 117.4603] & 112.8881 & 9.1444 & 4.5722 \\
\hline & {$\left[\beta_{3}^{\alpha}\right]$} & {$[137.8346$} & $148.0568]$ & 142.9457 & 10.2222 & 5.1111 \\
\hline & {$\left[\beta_{4}^{\alpha}\right]$} & {$[64.7554$} & $74.0221]$ & 69.3887 & 9.2667 & 4.6333 \\
\hline & {$\left[\beta_{5}^{\alpha}\right]$} & [71..513 & 82.263] & 76.8880 & 10.7500 & 5.3750 \\
\hline & {$\left[\beta_{6}^{\alpha}\right]$} & {$[44.7326$} & 54.8909] & 49.8117 & 10.1583 & 5.0792 \\
\hline \multirow{6}{*}{0.95} & {$\left[\beta_{0}^{\alpha}\right]$} & {$[323.2297$} & 327.7945] & 325.5121 & 4.5648 & 2.2824 \\
\hline & {$\left[\beta_{1}^{\alpha}\right]$} & {$[177.0718$} & 181.8107] & 179.4413 & 4.7389 & 2.3694 \\
\hline & {$\left[\beta_{2}^{\alpha}\right]$} & {$[110.7809$} & $115.3531]$ & 113.0670 & 4.5722 & 2.2861 \\
\hline & {$\left[\beta_{3}^{\alpha}\right]$} & {$[140.7298$} & 145.8409] & 143.2853 & 5.1111 & 2.5556 \\
\hline & {$\left[\beta_{4}^{\alpha}\right]$} & {$[67.1724$} & 71.8057] & 69.4890 & 4.6333 & 2.3167 \\
\hline & {$\left[\beta_{5}^{\alpha}\right]$} & {$[74.2683$} & 79.6433] & 76.9558 & 5.3750 & 2.6875 \\
\hline
\end{tabular}




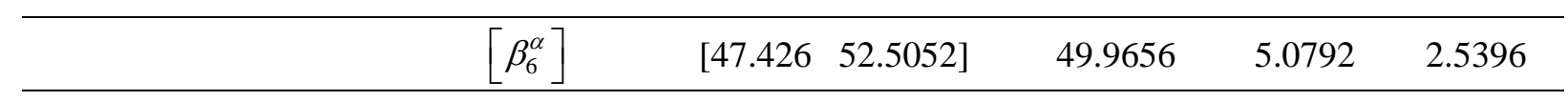

The Hausdorff metric calculation results are presented in Table 9.

Table 9. Hausdorff metric of interval type parameter estimates Printing Ink data set

\begin{tabular}{ccccccccc}
\hline $\begin{array}{c}\text { alpha-cut } \\
\text { levels }\end{array}$ & $H_{1}$ & $H_{2}$ & $H_{3}$ & $H_{4}$ & $H_{5}$ & $H_{6}$ & $H_{7}$ & $H_{8}$ \\
\hline $\mathbf{0}$ & 6.4436 & $\mathbf{1 . 3 7 5 1}$ & $\mathbf{3 . 2 8 2 4}$ & 7.7175 & $\mathbf{1 4 . 9 4 5 6}$ & $\mathbf{6 . 1 6 7 9}$ & $\mathbf{1 2 . 3 9 0 1}$ & $\mathbf{5 2 . 3 2 2 2}$ \\
$\mathbf{0 . 0 5}$ & 4.6999 & 3.8730 & 5.7475 & $\mathbf{5 . 9 1 8 4}$ & 17.3626 & 8.9232 & 15.0836 & 61.6082 \\
$\mathbf{0 . 1 0}$ & $\mathbf{2 . 9 5 6 1}$ & 6.3710 & 8.2126 & 8.8135 & 19.7795 & 11.6786 & 17.7770 & 75.5883 \\
$\mathbf{0 . 2 0}$ & 6.0628 & 11.3668 & 13.1428 & 14.6038 & 24.6135 & 17.1893 & 23.1640 & 110.1430 \\
$\mathbf{0 . 3 0}$ & 11.7049 & 16.3627 & 18.0729 & 20.3940 & 29.4474 & 22.7000 & 28.5510 & 147.2329 \\
$\mathbf{0 . 4 0}$ & 17.3470 & 21.3586 & 23.0031 & 26.1843 & 34.2813 & 28.2107 & 33.9379 & 184.3229 \\
$\mathbf{0 . 5 0}$ & 22.9891 & 26.3544 & 27.9332 & 31.9746 & 39.1153 & 33.7214 & 39.3248 & 221.4128 \\
$\mathbf{0 . 6 0}$ & 28.6312 & 31.3503 & 32.8634 & 37.7648 & 43.9492 & 39.2321 & 44.7118 & 258.5028 \\
$\mathbf{0 . 7 0}$ & 34.2733 & 36.3462 & 37.7935 & 43.5551 & 48.7831 & 44.7428 & 50.0987 & 295.5927 \\
$\mathbf{0 . 8 0}$ & 39.9154 & 41.3420 & 42.7237 & 49.3454 & 53.6171 & 50.2535 & 55.4857 & 332.6828 \\
$\mathbf{0 . 9 0}$ & 45.5575 & 46.3379 & 47.6538 & 55.1356 & 58.4510 & 55.7642 & 60.8727 & 369.7727 \\
$\mathbf{0 . 9 5}$ & 48.3786 & 48.8358 & 50.1189 & 58.0308 & 60.8680 & 58.5195 & 63.5661 & 388.3177 \\
\hline
\end{tabular}

It can be easily seen from Table 9 that the similarity of fuzzy and Bayesian model parameter estimates are high at $\alpha \in[0,0.10]$ - cut levels according to the $H$ metric. From Table 9, it is possible to prefer fuzzy modeling approach for $\alpha \in[0,0.10]$ - cut levels alternatively modeling with Bayesian approach for interval type parameter estimates.

\section{CONCLUSION}

This study presents the alternatively preferability of possibilistic modeling approach to probabilistic modeling approach for RRM data sets. For this purpose, fuzzy and Bayesian linear modeling approaches are applied to RRM data sets. After obtaining model parameter estimates, it is aimed to compare the uncertainty of model parameters. Therefore, it is needed to define the estimated values of parameters on the same domain. This is achieved with interval type presentation of parameter estimates through alpha-cut level intervals and HPD credible intervals for fuzzy and Bayesian approaches, respectively. mid $([\boldsymbol{\beta}])$, $w([\boldsymbol{\beta}]), \operatorname{rad}([\boldsymbol{\beta}])$ and $H$ metrics of intervals are used as comparison metric tools for both deterministic closed intervals of fuzzy and Bayesian models. It should be noted here that the comparison is achieved between Bayesian interval type estimates with fuzzy interval type estimates for each defined alpha-cut levels, $\alpha \in[0,1]$. It is seen from the results that the $H$ metric values of interval type estimates are roughly similar for $\alpha \in[0.20,0.30]$-cut and $\alpha \in[0,0.10]$-cut levels of fuzzy approach with $95 \%$ confident of Bayesian approach for Roman Catapult data set and Printing Ink data set, respectively. Thus, someone can prefer to use fuzzy linear modeling approach instead of Bayesian to model the RRM data set and to analyse the uncertainty of unknown model parameters with great flexibility and without any strict modeling assumptions. It is possible to say that the fuzzy modeling could be directly applied to RRM data set with $\alpha \in[0,0.30]$-cut levels without making comparison analysis with Bayesian approach. For future work, different types of fuzzy numbers and different confidence levels are planned to apply to the RRM data set for fuzzy modeling and Bayesian modeling, respectively. 


\section{CONFLICTS OF INTEREST}

No conflict of interest was declared by the author.

\section{REFERENCES}

[1] Khuri, A. I., Mukhopadhyay, S., "Response surface methodology", WIREs Computational Statistics, $2: 128-149,(2010)$.

[2] Zadeh, L. A., "The concept of a Linguistic variable and applications to approximate reasoning-part-I, II, III”, Information Science, 8:199-249, (1975).

[3] Lai, Y. J., Chang, S., "A fuzzy approach for multi response optimization: An off-line quality engineering problem”, Fuzzy Sets and Systems, 63:117-129, (1994).

[4] Akbar, M. S., Otok, B. W., Prastyo, D. D., "Fuzzy modeling approach and global optimization for dual response surface", Jurnal Teknik Industri, 9:102-111, (2007)

[5] Xie, H., Lee, Y. C., "Process optimization using a fuzzy logic response surface method", IEEE Transactions on Components, Packaging, and Manufacturing Technology-Part A, 17 (1994).

[6] Prasad, K., Nath, N., "Comparison of sugarcane juice based beverage optimisation using response surface methodology with fuzzy method", Sugar Tech., 4:109-115, (2002).

[7] Xu, R., Dong, Z., "Fuzzy modeling in response surface method for complex computer model based design optimization", Proc. 2nd IEEE/ASME Int. Conf. Mechatronic and Embedded Systems and Applications, 1-6, (2006).

[8] Bashiri, M., Hosseininezhad, S. J., "A fuzzy programming for optimizing multi-response surface in robust designs", Journal of Uncertain Systems, 3:163-173, (2009).

[9] Bashiri, M., Hosseininezhad, S. J., "Fuzzy development of multiple response optimization", Group Decision and Negotiation 21:417-438, (2012).

[10] Türkşen, Ö., Güler, N., "Comparison of fuzzy logic based models for the multi-response surface problems with replicated response measures", Applied Soft Computing, 37:887-896, (2015).

[11] Türkşen, Ö., "Analysis of Response Surface Model Parameters with Bayesian Approach and Fuzzy Approach", International Journal of Uncertainty, Fuzziness and Knowledge-Based Systems, 24:109122, (2016).

[12] Türkşen, Ö., "A Fuzzy Modeling Approach for Replicated Response Measures Based on Fuzzification of Replications with Descriptive Statistics and Golden Ratio", Süleyman Demirel University Journal of Natural and Applied Sciences, 22:153-159, (2018).

[13] Steinberg, D. M., "A Bayesian Approach to Flexible Modeling of Multivariable Response Functions", Journal of Multivariate Analysis, 34:157-172, (1990).

[14] Chen, Y., "Bayesian Hierarchical Modelling of Dual Response Surfaces", Ph.D. thesis, Virginia Polytechnic Institute and State University, (2005).

[15] Chen, Y., Ye, K., "Bayesian Hierarchical Modelling on Dual Response Surfaces in Partially Replicated Designs”, Quality Technology \& Quantitative Management, 6:371-389, (2009). 
[16] Chen, Y., Ye, K., “A Bayesian hierarchical approach to dual response surface modelling”, Journal of Applied Statistics, 38:1963-1975, (2011).

[17] Peterson, J. J., Quesada, G. M., Castillo, E., "A Bayesian Reliability Approach to Multiple Response Optimization with Seemingly Unrelated Regression Models", Quality Technology \& Quantitative Management, 6:353-369, (2009).

[18] Quesada, G. M., Castillo, E., Peterson, J. J., "A Bayesian Approach for Multiple Response Surface Optimization in the Presence of Noise Variables", 1-24, (2002).

[19] Kozan, E., Köksoy, O., “A Bayesian Parameter Estimation Approach to Response Surface Optimization in Quality Engineering", Sakarya University Journal of Science, 23:767-774, (2019).

[20] Wang, L., Xiong, C., Yang, Y., "A novel methodology of reliability-based multidisciplimary design optimization under hybrid interval and fuzzy uncertainties", Computer Methods in Applied Mechanics and Engineering, 337:439-457, (2018).

[21] Wang, L., Xiong, C., Wang, X., Xu, M., Li, Y., “A dimesion-wise method and its improvement for multidisciplinary interval uncertainty analysis", Applied Mathematical Modelling, 59:680-695, (2018).

[22] Wang, L., Wang, X., Su, H., Lin, G., "Reliability estimation of fatigue crack growth prediction via limited measured data", International Journal of Mechanical Sciences, 121:44-57, (2017).

[23] Moore, R. E., Kearfott, R. B., Cloud, M. J., "Introduction to Interval Analysis”, SIAM, (2009).

[24] Palumbo, F., Irpino, A. "Multidimensional Interval-Data: Metrics and Factorial Analysis", Multivariate Statistical and Visualization Methods to Analyze, to Summarize, and to Evaluate Performance Indicators, 689-698, (2005).

[25] Diamond, P. "Fuzzy least squares”, Information Sciences, 46:141-157, (1998).

[26] Paulino, C. D., Turkman, M. A. A., Murteira, B., Silva, G. L. "Estatistica Bayesiana”, Fundaçao Calouste Gulbenkian, (2018).

[27] Chachi, J., Taheri, S. M. "A Least-Absolutes Regression Model for Imprecise Response Based on the Generalized Hausdorff- Metric”, Journal of Uncertain Systems, 7:265-276, (2013).

[28] Galdino, S. "Interval-valued Data Clustering Based on the Range City Block Metric", 2016 IEEE International Conference on Systems, Man, and Cybernetics-SMC 2016, 228-234, (2016).

[29] Luner, J.J. "Achieving continuous improvement with the dual response approach: A demonstration of the Roman catapult", Quality Engineering ,6:691-705, (1994).

[30] Box, G.E.P., Draper, N.R. "Response Surface Mixtures and Ridge Analysis", John Wiley and Sons, (2007). 